\title{
Atorvastatin in stroke: a review of SPARCL and subgroup analysis
}

\author{
This article was published in the following Dove Press journal: \\ Vascular Health and Risk Management \\ 27 March 2010 \\ Number of times this article has been viewed
}

\author{
Branko $\mathrm{N}$ Huisa \\ Andrew B Stemer \\ Justin A Zivin \\ Department of Neuroscience \\ University of California, San Diego, \\ CA, USA
}

\begin{abstract}
Statin therapy in patients with cardiovascular disease is associated with reduced incidence of stroke. The Stroke Prevention by Aggressive Reduction of Cholesterol Levels (SPARCL) trial showed daily treatment with $80 \mathrm{mg}$ of atorvastatin in patients with a recent stroke or transient ischemic attack (TIA) reduced the incidence of fatal or nonfatal stroke by $16 \%$. Several post hoc analyses of different subgroups followed the SPARCL study. They have not revealed any significant differences when patients were sorted by age, sex, presence of carotid disease or type of stroke, with the exception of intracranial hemorrhage as the entry event. Lower low-density lipoprotein cholesterol levels in addition to possible neuroprotective mechanisms due to atorvastatin treatment correlate with improved risk reduction. Although not predefined subgroups and subject to an insufficient power, these post hoc studies have generated new clinical questions. However, clinicians should avoid denying therapy based on such subgroup analysis. At this point, the best evidence powerfully demonstrates stroke and TIA patients should be prescribed high dose statin therapy for secondary stroke prevention.
\end{abstract}

Keywords: statins, intracranial hemorrhage, neuroprotection, outcome, prevention, carotid stenosis, transient ischemic attack

\section{Introduction}

Statins, as 3-hydroxy-3-methylglutaryl coenzyme-A (HMG-CoA) reductase inhibitors, belong to a well established class of drugs that can reduce cholesterol, ameliorate vascular atherosclerosis and improve cardiovascular morbidity and mortality. ${ }^{1-5}$ Previous clinical trials demonstrated that statins reduce the first or recurrent stroke risk among patients with known heart disease and subsequently have become cornerstones of therapy for secondary prevention of vascular disease along with antiplatelets and antihypertensives. ${ }^{6}$ Among the statins atorvastatin is a synthetic type of HMG-CoA analogue that exhibits a substantial efficacy for decreasing total and low-density lipoprotein cholesterol (LDL-C) levels, triglycerides and modification of lipoprotein composition. The Stroke Prevention by Aggressive Reduction in Cholesterol Levels (SPARCL) trial was the only study to test whether high doses of atorvastatin would reduce the risk of secondary stroke in patients with a previous stroke or transient ischemic attack (TIA) but without known heart disease. ${ }^{7}$ This paper will review the SPARCL trial, its sub group analyses, and related studies.

\section{Mechanism of action}

The beneficial effects of statins result, at least in part, from direct inhibition of HMG-CoA reductase. ${ }^{8}$ This inhibition reduces endogenous cholesterol biosynthesis 
and increases the expression of LDL receptors responsible for LDL-C uptake and clearance. ${ }^{8}$ These do not seem to be the only mechanisms of action, and several other so-called "pleotropic" effects have been suggested. ${ }^{8}$ Those beneficial effects include modification of endothelial function, reduction of inflammatory responses, increase in plaque stability, and inhibition of platelets with decreased thrombus formation. However, the neuroprotective mechanisms of statins against stroke are not well understood. Stroke animal models suggested that augmentation of cerebral blood flow by increasing nitric oxygen (NO) production, decrease of glutamate excitotoxicity, neurogenesis and angiogenesis are responsible for some of its neuroprotective actions. ${ }^{9-14}$ So far, the clinical importance of these nonlipid lowering properties remains uncertain.

\section{The SPARCL trial}

SPARCL was a prospective, double-blind, placebo-controlled international trial conducted at 205 centers, in which 4731 patients with a history of stroke or TIA were randomized. Patients received either atorvastatin $80 \mathrm{mg}$ per day $(n=2365)$ or placebo $(n=2366)$ and were followed for an average of 4.9 years. ${ }^{7}$ Stroke, or a TIA, was diagnosed by a neurologist within 30 days and randomization occurred within 1 to 6 months after the event. LDL-C concentration required being between 100 and $190 \mathrm{mg} / \mathrm{dL}$, and any lipidaltering treatments had to be discontinued 30 days before the first screening visit. Patients with hemorrhagic stroke were included only if they were believed to be at risk for ischemic stroke or coronary artery disease; patients with atrial fibrillation, cardiac sources of embolism and subarachnoid hemorrhage were excluded. The primary outcome was any nonfatal or fatal stroke after randomization. The analysis plan was prespecified and performed on an intention to treat basis with the inclusion of all patients who underwent randomization.

Demographics, mean LDL-C, stroke type, concomitant therapy, stroke risk factors and history of prior statin therapy were evenly distributed between placebo and treatment groups. The primary study endpoint, fatal or nonfatal stroke, was significantly less frequent in the atorvastatin group $(11.2 \%)$ versus placebo $(13.1 \%)$ and represented a relative

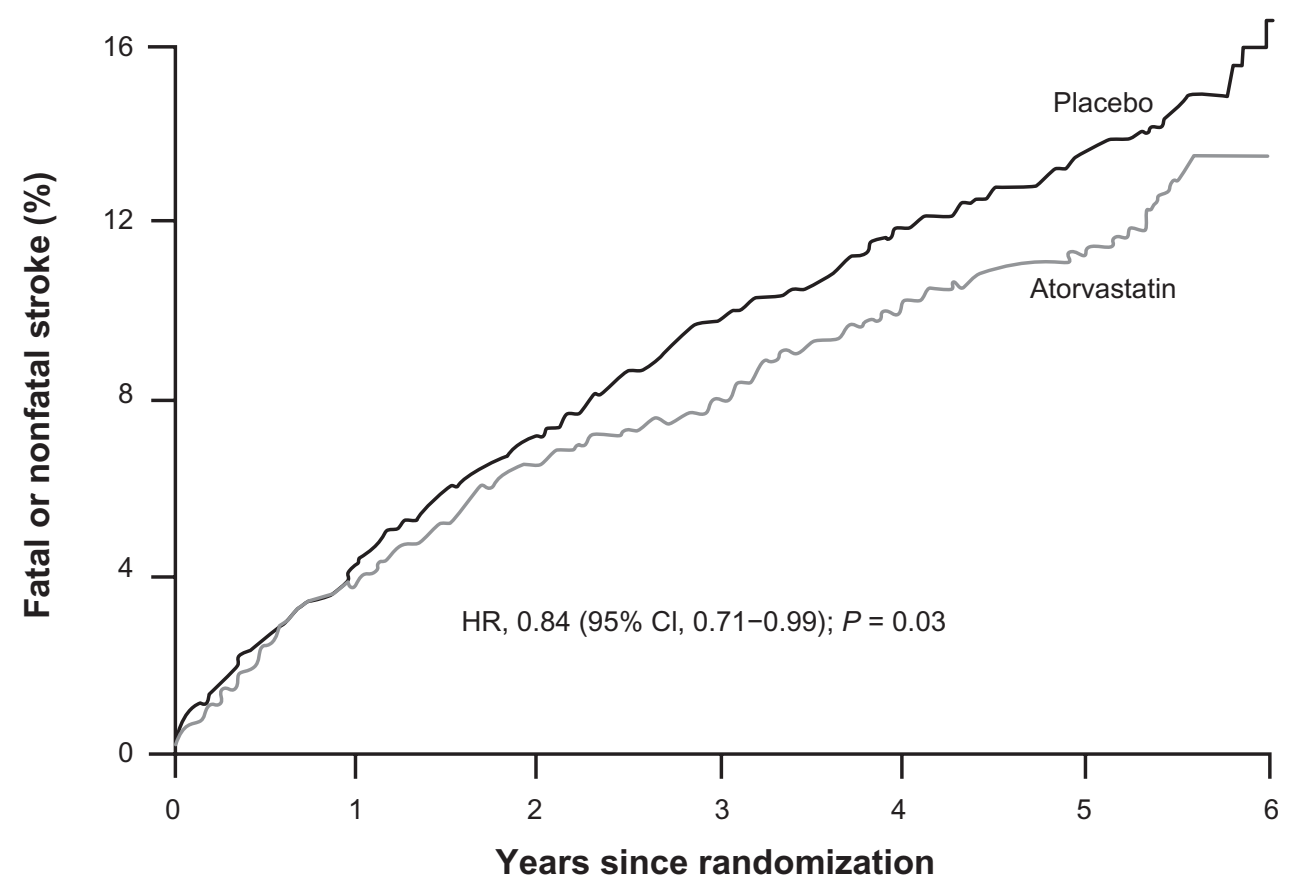

\section{No at risk}

Atorvastatin

2365

2208

2106

2031

1935

922

126

Placebo

2366

2213

2115

2010

1926

887

137

Figure I Kaplan-Meier curves for the first occurrence of primary endpoint (fatal or nonfatal stroke).

Reproduced with permission from The Stroke Prevention by Aggressive Reduction in Cholesterol Levels (SPARCL) Investigators. High-dose atorvastatin after stroke or transient ischemic attack. N Engl J Med. 2006;355:549-559.7 Copyright (C) 2006 Massachusetts Medical Society. All rights reserved.

Abbreviations: $\mathrm{HR}$, hazard ratio; $\mathrm{Cl}$, confidence interval. 
risk reduction $(\mathrm{RRR})$ of $16 \%(P=0.03,95 \%$ confidence interval [CI]; 0.71-0.99) (Figure 1). Interestingly, this effect was driven predominately by reduced adjusted relative risk of fatal stroke which was decreased by $43 \%(P=0.03)$, whereas atorvastatin had no significant effect on nonfatal stroke reduction $(P=0.11)^{7}$

Every secondary endpoint showed significant improvement with atorvastatin treatment: reduced relative risks of stroke and TIA (23\%; $P<0.001)$, TIA alone (26\%; $P=0.004)$, major coronary events $(35 \% ; P=0.003)$, nonfatal myocardial infarction ( $49 \% ; P=0.001)$, major cardiovascular events (20\%; $P=0.002)$, acute coronary events $(35 \% ; P=0.001)$, any coronary events $(42 \% ; P<0.001)$, revascularization procedures $(45 \% ; P<0.001)$, and any cardiovascular event $(26 \% ; P<0.001) .{ }^{7}$ Total mortality $(9.1 \%$ vs $8.9 \%)$, and cancer related mortality ( $2.4 \%$ vs $2.2 \%)$ did not significantly differ between groups.

After randomization, more patients in the placebo group withdrew consent $(P=0.07)$ or permanently stopped the study medication $(P=0.07)$. Approximately half as many atorvastatin (compared to placebo) recipients received openlabel statin therapy ( $11.4 \%$ vs $25.4 \%)$, and, in both study groups, atorvastatin was the most widely used nonstudy open-label statin. ${ }^{7}$ The use of open label statins reduced the frequency of strokes in the placebo group; therefore the overall effect size appeared to be smaller than it actually was.

In contrast to the reduction of ischemic stroke and TIA, there was a significant increase in the incidence of hemorrhagic stroke in the atorvastatin group $(2.3 \%$ vs $1.4 \%)$. Regarding adverse effects, a benign but significant elevation of aspartate aminotransferase occurred in the treatment group but was not associated with liver failure or rhabdomyolisis. No other differences in adverse events and laboratory values were noted.

With a number needed to treat (NNT) of 143 patients to prevent one recurrent ischemic stroke per year, aspirin is considered a first line medical therapy for secondary stroke prevention. ${ }^{15}$ In comparison, the NNT with atorvastatin to prevent one stroke is 46 patients over 5 years and is likely much lower due to poor adherence in the treatment group. While the benefit of atorvastatin may not be apparent to every individual, it has an enormous impact when applied to an entire population. Furthermore, the reduction of cardiac and peripheral arterial disease in this group is important. In summary, SPARCL demonstrated that high dose atorvastatin decreased the risk of secondary stroke, major coronary events and revascularization procedures among patients with a recent stroke or TIA. ${ }^{7}$

\section{SPARCL subgroup analysis}

Multiple publications consisting of subgroup analyses originated from SPARCL. Although not predefined in the original study and inadequately powered, these post hoc studies suggest answers to important clinical questions, generate new hypothesis and strengthen (or weaken) previous theories of statin use in stroke patients. However, any subgroup analysis should be interpreted cautiously until further confirmatory studies are performed.

\section{Intracranial hemorrhage (ICH)}

Epidemiological and observational studies have shown a relationship between low cholesterol levels and hemorrhagic stroke but that difference was not found in trials with statins given for coronary artery disease. ${ }^{16-19}$ In SPARCL, the overall incidence of hemorrhagic stroke was low $(1.8 \%){ }^{7}$ While mortality from hemorrhagic stroke was similar (17 in the atorvastatin vs 18 patients in the placebo group) there was a statistically significant difference between the two groups $(2.3 \%$ in the atorvastatin vs $1.4 \%$ in the placebo group; $P=0.01){ }^{7}$ Post hoc analyses based on stroke type revealed that atorvastatin reduced the relative risk of fatal and not fatal of ischemic stroke by $21 \%(P=0.01)$; this effect was partially attenuated by an increased risk of hemorrhage (unadjusted hazard ratio [HR] $0.79,95 \%$ CI 1.09-2.59) resulting in the overall reduction of $16 \%$ in the risk of fatal and nonfatal stroke. ${ }^{20}$ Interestingly, the risk of hemorrhagic stroke was not related to of LDL-C levels in statin-treated subjects. Using Cox multivariable regression analysis male sex, hypertension, advancing age and a hemorrhagic or small vessel stroke upon study entry were independent risk factors associated with hemorrhagic stroke. ${ }^{20}$ Treatment did not disproportionally increase the hemorrhage risk in these patients; conversely, those with hemorrhagic stroke at entry did not benefit from treatment (HR 2.82, 95\% CI 0.89-9.01). ${ }^{20}$ The SPARCL findings contradict several cardiac interventional studies using statins where increased intracranial hemorrhage was not found. ${ }^{1-4}$ In the Heart Protection Study, patients enrolled with prior history of stroke were found to have a nonsignificant increase in hemorrhagic stroke if treated with simvastatin $40 \mathrm{mg}$ per day compared with the placebo group. ${ }^{5}$ Because most SPARCL patients had stroke as entry event $(60.9 \%)$, it is possible that patients with prior cerebrovascular accident are prone to ICH after statin therapy. Based on the SPARCL data, it seems that patients with hemorrhagic stroke as an entry event may not benefit from 
statin therapy. Most importantly though, as the authors conclude, the theoretical risk of ICH with high dose statins should be balanced with the compelling benefit of treatment that reduces the overall risk of stroke and other cardiovascular events.

\section{LDL-C and outcome in SPARCL}

LDL-C reduction was used as a marker for adherence to the allocated treatment and patients were subsequently classified into levels of LDL-C reduction. Based on 55,045 blinded measures, patients with $\geq 50 \%$ reduction had a $31 \%$ decrease in the combined risk of fatal and nonfatal stroke. ${ }^{21}$ This was approximately twice the $16 \%$ observed in the prespecified intention to treat analysis (Figure 2). ${ }^{7}$ Additionally, increased ICH were not observed in the group with the greatest reduction of LDL-C. ${ }^{21}$ Only lower high-density lipoprotein cholesterol (HDL-C) levels at baseline predicted the risk of both, recurrent stroke and mayor cardiovascular events; however, high baseline levels of triglycerides and LDL/HDL ratio were associated to the occurrence of mayor cardiovascular events. ${ }^{22}$ In the treatment group, higher HDL-C levels were maintained at low levels of LDL-C and a there was cumulative protective effect for major cardiovascular events and stroke when having all together, lower LDC-L, higher HDL-C and optimal blood pressure control. ${ }^{23}$ Although the intention to treat analysis is often the best way to determine treatment efficacy, this post hoc analysis using LDL-C levels as a surrogate of high dose atorvastatin, attempts to answer the initial SPARCL hypothesis disregarding noncompliance observed during trial. The findings are consistent with two previous metaanalysis revealing an association between reduced stroke risk and reduction of LDL-C. ${ }^{6,24}$ However, because the relationship between stroke and cholesterol levels is not as clearly defined as for coronary artery disease, the doserelated stroke risk reduction found in this analysis could be attributed to pleotropic effects and vascular protection rather than simple LDL-C reduction.

\section{PRIMARY END POINT}

All atorvastatin patients

$\geq 50 \%$ decrease of C-LDL

$<50 \%$ decrease of C-LDL

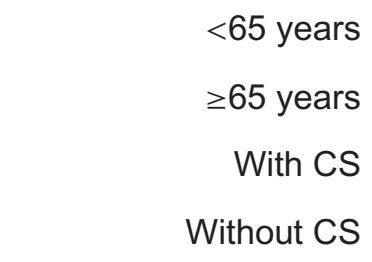

Small vessel disease*

Large vessel*

TIA*

Hemorrhagic*

Unknown stroke type*

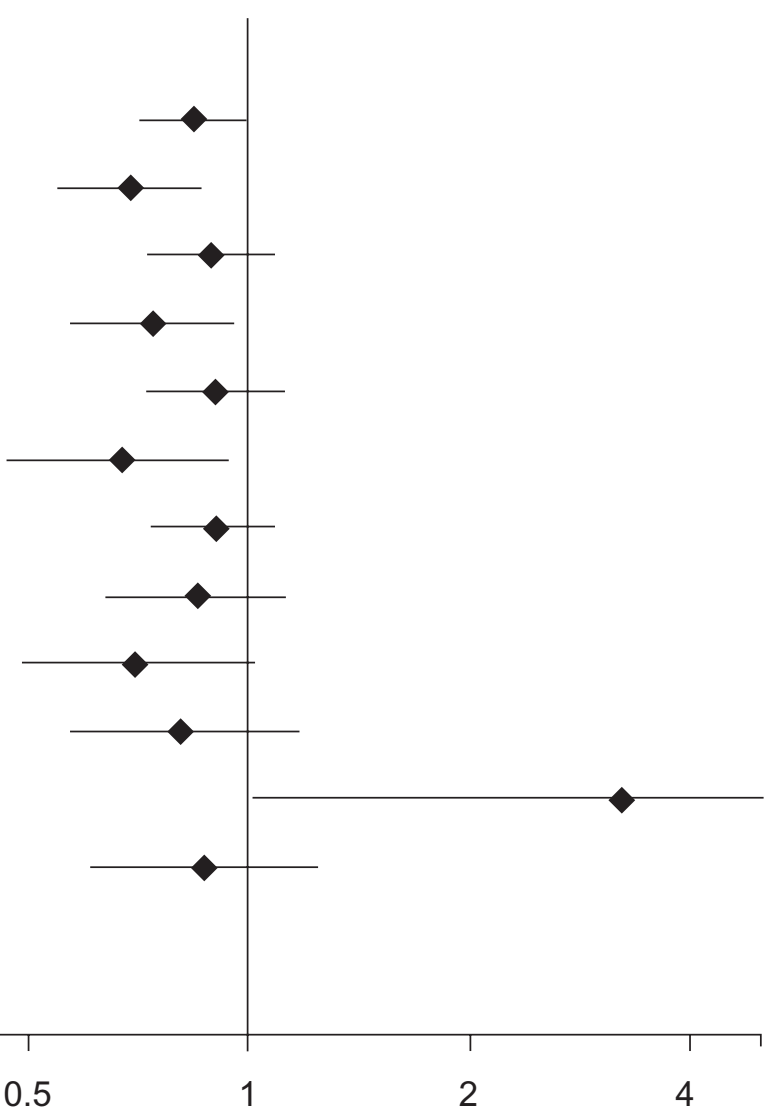

Hazard ratio $(95 \% \mathrm{Cl})$

Figure 2 Primary endpoint (fatal or nonfatal stroke) by different patient subgroups showing the treatment hazard ratios from the Cox regression model.

*Type of event at entry study.

Abbreviations: CS, carotid stenosis; Cl, confidence interval; C-LDL, low-density lipoprotein cholesterol; TIA, transient ischemic attack. 


\section{Age and sex differences in SPARCL}

Stroke is more frequent in individuals 65 years or older. ${ }^{25,26}$ Several studies have shown the benefits of statin treatment in elderly patients, particularly those with established vascular disease or risk factors for it. ${ }^{5,27-30}$ Unfortunately, elderly patients are less likely to be prescribed statins, and have worse compliance compared to younger patients..$^{31} \mathrm{An}$ analysis of the entire SPARCL cohort, using an intention to treat protocol, compared primary and secondary endpoints between patients over 65 and under 65 years old. ${ }^{32}$ Patients over 65 were more likely to have hypertension, a history of carotid stenosis at baseline, and less likely to be current smokers compared with younger patients. ${ }^{32}$ For the primary endpoint there was a $10 \%$ RRR (HR 0.90, 0.73-1.11, $P=0.33$ ) in the elderly group compared to a $26 \%$ RRR in the younger group (HR 0.74, 0.57-0.96, $P=0.02$ ). ${ }^{32}$ This corresponded to an absolute risk reduction of $1.5 \%$ in the elderly group and $2.6 \%$ in the younger group (Figure 2)..$^{32} \mathrm{~A}$ test of heterogeneity for a treatment-age interaction, however, was not significant $(P=0.52) .{ }^{32}$ There were no treatment interactions associated with age for the SPARCL primary endpoint or any of the SPARCL secondary endpoints. ${ }^{32}$ Atorvastatin was well tolerated in both groups, though serious adverse events were more common in the elderly with either active treatment or placebo. ${ }^{32}$ Significant elevation in liver enzymes and myopathy were uncommon with atorvastatin and equally reported in both the cohorts. ${ }^{32}$ Although the risk reduction with high dose statins was not significant for older patients, no interactions were found between age and treatment effect. The authors note the study was not powered for detecting risk reduction in older patients. Ultimately, all we can conclude is the findings suggest a possible benefit in stroke prevention in the elderly group.

Regarding gender, a secondary analysis of the effects of statin therapy in primary and secondary endpoints in men and women was also attempted. ${ }^{33}$ Although stroke risk factor profiles from the SPARCL baseline data differed for men and women, a secondary analysis did not find any differences in statin treatment effects or adverse reactions. ${ }^{33}$

\section{SPARCL by stroke subtypes}

Differences in outcomes between various stroke subtypes were also examined. Baseline stroke subtype assignment was based on local investigator judgment and not standardized or adjudicated. Neuroimaging data was not available for the final analysis but it was used by local investigators for the elucidation of stroke subtype. Among 4731 participants, 4728 had information regarding entry event subtype with
$15.8 \%$ classified as having large vessel disease, $29.8 \%$ small vessel disease, $21.5 \%$ ischemic stroke of unknown cause, $30.9 \%$ TIA, and $2 \%$ hemorrhagic stroke. ${ }^{34}$ Primary and secondary endpoints were obtained and no differences were found across baseline stroke subtypes with the exception of hemorrhagic stroke (Figure 2) ${ }^{34}$ This post hoc analysis was exploratory as it oversimplified stroke subtypes and lacked power for subgroup analyses. It contained an estimated a power of $51 \%$ to detect the risk reduction of $16 \%$ observed for the primary SPARCL end point between all entry event stroke subtypes. The power to detect a risk reduction of $16 \%$ was $20 \%$ for the small vessel disease group but only $6 \%$ for the hemorrhagic stroke group. Hence, a difference among ischemic stroke subtypes may exist but could not be proven by this subgroup analysis.

\section{Atorvastatin and carotid disease}

Carotid artery evaluation was not required by the SPARCL protocol, but 4278 (90.4\%) of the SPARCL subjects underwent carotid imaging by the local investigators at the time of patient randomization. ${ }^{35} 3724$ subjects did not have carotid stenosis while 1007 subjects had carotid stenosis, including 453 subjects categorized as unknown carotid status. ${ }^{35}$ Within the carotid stenosis group, a primary end point occurred in 55 patients randomized to atorvastatin and in 83 randomized to placebo. ${ }^{35}$ Patients with carotid stenosis randomized to atorvastatin reduced their risk of stroke by $33 \%$ (HR 0.67, 95\% CI $0.47-0.94 ; P=0.02$ ) (Figure 2) and risk of TIA or stroke by $34 \%$ (HR $0.66,95 \%$ CI $0.50-0.89 ; P=0.005) .{ }^{35}$ Furthermore, all cardiovascular end points were reduced in patients with carotid stenosis treated with atorvastatin: major coronary events by $43 \%$ (HR $0.57,95 \%$ CI $0.32-1.00 ; P=0.05$ ), any cardiovascular event by $42 \%$ (HR $0.58,95 \%$ CI $0.46-0.73$; $P<0.0001$ ), any revascularization procedure by $51 \%$ (HR $0.49,95 \%$ CI $0.33-0.73 ; P=0.0004)$, and carotid revascularizations by $56 \%$ (HR $0.44,95 \%$ CI $0.24-0.79 ; P=0.006$ ). ${ }^{35}$ With the exception of carotid revascularization there was not heterogeneity regarding primary and secondary endpoints. However, the carotid stenosis group treated with atorvastatin had a higher absolute risk reduction and benefited more when all cardiovascular events were taken into consideration. ${ }^{35}$

The authors estimated a NNT of 20 patients treated for 5 years to prevent one stroke using high dose atorvastatin in patients with carotid disease. Considering all cardiovascular events, the annual risk reduction exceeded $2.5 \%$ per year estimating a NNT of 8 patients over 5 years. This is comparable to performing carotid endarterectomy in asymptomatic patients but without the risks of surgery or additional 
benefits of reduced cardiovascular events. ${ }^{36,37}$ This subanalysis suggested that patients with carotid disease receive the most benefit for secondary stroke prevention. This implies that statins powerfully modulate atherosclerotic disease and could, in theory, similarly benefit patients with aortic arch or intracranial stenosis.

\section{Statin use and stroke outcome}

There is ample laboratory data suggesting pleotropic effects of statins might not only affect the risk of stroke but also secondary stroke outcome. ${ }^{8}$ In the SPARCL trial a total of 265 patients randomized to atorvastatin and 311 patients randomized to placebo suffered a stroke. ${ }^{7}$ An exploratory analysis of the trial assessed whether treatment favorably changed the distribution of stroke severities using the modified Rankin scale (mRS), National Institute of Health Stroke Scale (NISSS) and Bathel Index (BI) at enrollment and 90 days post stroke. ${ }^{38}$ Among all subjects having an ischemic stroke during the trial, there was a trend toward less severe outcomes in those taking atorvastatin based on the mRS in $(P=0.647)$ (Figure 3). This was true despite poor adherence to randomized treatments. No other differences were significant. The authors found a significant reduction of all stroke events and all ischemic events $(P<0.001)$ using Mantel-Haenszel test after creating a composite of fatal stroke, severe (mRS 5-4), moderate (mRS 3-2), mild (mRS 1-0), TIA and no events. ${ }^{38}$ However, this analysis combined stroke prevention and stroke "attenuation" properties making it inadequate for definitively determining a favorable outcome after a secondary stroke. Possible reduction in secondary stroke severity using statins has been suggested by observational studies but has yet to be proven. ${ }^{39-42}$ Interestingly, there is experimental animal ${ }^{43,44}$ and human epidemiological data ${ }^{45,46}$ revealing that prior use of statins protects the brain and improves outcomes after intracranial hemorrhage. The latter was not replicated by the SPACRL trial.

\section{Stroke as a coronary risk equivalent}

During the 4.9 years of follow-up in SPARCL, patients with recent stroke or TIA had a $5.1 \%$ incidence of a major coronary event (death from cardiac causes [1.6\%], nonfatal myocardial infarction [3.5\%]), major cardiovascular event (17.2\%), any coronary event (8.6\%) and need for revascularization (coronary, carotid or peripheral; 6.9\%). ${ }^{7}$ High dose atorvastatin reduced major coronary events by $35 \%$ (HR 0.65,CI 95\% $0.49-0.87$ ), any coronary event by $42 \%$ (HR $0.58,95 \% \mathrm{CI}$ $0.46-0.73])$ and the need for revascularization by $45 \%$ (HR $0.55,95 \%$ CI $0.43-0.72){ }^{7}$ The high incidence of coronary vascular events in patients with ischemic stroke, as well as the profound reduction of coronary events with statin treatment, has caused stroke and TIA to be considered a coronary risk equivalent. ${ }^{47}$ SPARCL confirmed the high risk for nonstroke vascular events and their substantial reduction with atorvastatin. Recent reviews of clinical trials illustrate this relationship and suggest the idea of adding cardiovascular endpoint events in preventive stroke clinical trials. ${ }^{48,49}$ Future studies should assess the use of cardiac screening test within ischemic stroke patients.

\section{Conclusion}

The SPARCL trial has shown that high dose atorvastatin is an effective medication for secondary prevention of stroke

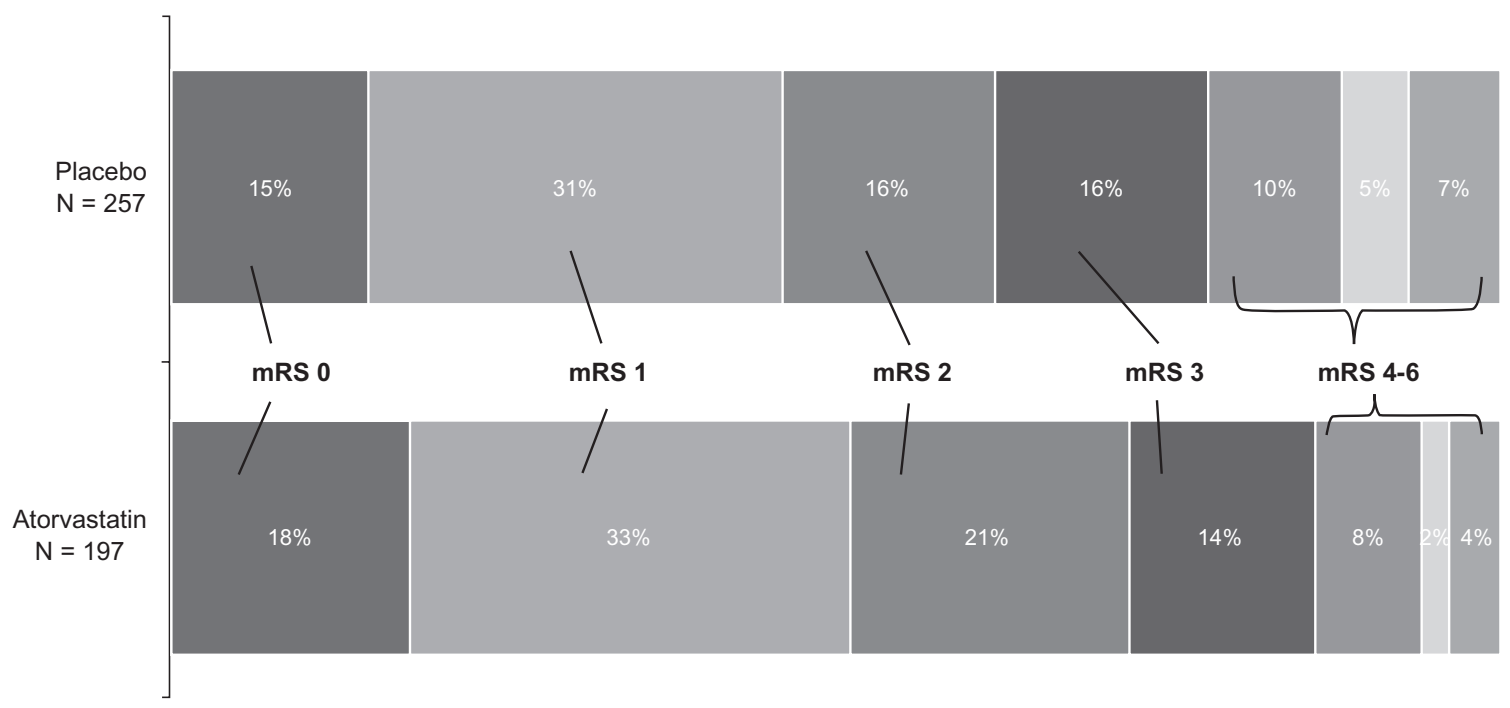

Figure 3 Stroke severity after 90 days measured by modified Rankin Score (mRS) from subjects who had only ischemic stroke during the trial. $P=0.067$ Using CochranMantel-Haenszel test.

From data of Goldstein et al. ${ }^{38}$ 
and cardiovascular events in patients with no known history of cardiac disease. ${ }^{7}$ Although treating patients with a history of ICH should be considered with caution, we feel it is inappropriate to deny an effective therapy to stroke patients based on subgroup analysis. Further studies might help to identify best responders and stratify risk groups.

\section{Acknowledgments}

The review was supported by the educational grant : NIH grant 3P50 NSO44148-07S2.

\section{Disclosures}

Dr Justin A Zivin is a paid consultant to Pfizer and steering committee member of the SPARCL trial. Dr Huisa and Dr Stemer have no disclosures.

\section{References}

1. Randomised trial of cholesterol lowering in 4444 patients with coronary heart disease: the Scandinavian Simvastatin Survival Study (4S). Lancet. 1994;344:1383-1389.

2. The Long-Term Intervention with Pravastatin in Ischaemic Disease (LIPID) Study Group. Prevention of cardiovascular events and death with pravastatin in patients with coronary heart disease and a broad range of initial cholesterol levels. N Engl J Med. 1998;339:1349-1357.

3. Plehn JF, Davis BR, Sacks FM, et al. Reduction of stroke incidence after myocardial infarction with pravastatin: the Cholesterol and Recurrent Events (CARE) study. Circulation. 1999;99:216-223.

4. Sever PS, Dahlof B, Poulter NR, et al. Prevention of coronary and stroke events with atorvastatin in hypertensive patients who have average or lower-than-average cholesterol concentrations, in the Anglo-Scandinavian Cardiac Outcomes Trial - Lipid Lowering Arm (ASCOT-LLA): a multicentre randomised controlled trial. Lancet. 2003;361:1149-1158.

5. Heart Protection Collaborative Study Group. MRC/BHF Heart Protection Study of cholesterol lowering with simvastatin in 20,536 high-risk individuals: a randomized placebo-controlled trial. Lancet. 2002;360:7-22.

6. Amarenco P, Labreuche J, Lavallée P, Touboul PJ. Statins in stroke prevention and carotid atherosclerosis: systematic review and metaanalysis. Stroke. 2004;35:2902-2909.

7. The Stroke Prevention by Aggressive Reduction in Cholesterol Levels (SPARCL) Investigators. High-dose atorvastatin after stroke or transient ischemic attack. N Engl J Med. 2006;355:549-559.

8. Liao JK. Effects of statins on 3-hydroxy-3-methylglutaryl coenzyme A reductase inhibition beyond low-density lipoprotein cholesterol. Am J Cardiol. 2005;96(Suppl):24F-33F.

9. Zhang L, Zhang ZG, Liu XS, Hozeska-Solgot A, Chopp M. The $\mathrm{PI} 3 \mathrm{~K} / \mathrm{Akt}$ pathway mediates the neuroprotective effect of atorvastatin in extending thrombolytic therapy after embolic stroke in the rat. Arterioscler Thromb Vasc Bio. 2007;27:2470-2475.

10. Chen JL, Zhang ZG, Li Y, et al. Statins induce angiogenesis, neurogenesis, and synaptogenesis after stroke. Ann Neurol. 2003;53:743-751.

11. Kawashima S, Yamashita T, Miwa Y, et al. HMG-CoA reductase inhibitor has protective effects against stroke events in stroke-prone spontaneously hypertensive rats. Stroke. 2003;34:157-163.

12. Lu D, Qu C, Goussev A, et al. Statins increase neurogenesis in the dentate gyrus, reduce delayed neuronal death in the hippocampal CA3 region, and improve spatial learning in rat after traumatic brain injury. J Neurotrauma. 2007;24:1132-1146.
13. Bosel J, Gandor F, Harms C, et al. Neuroprotective effects of atorvastatin against glutamate-induced excitotoxicity in primary cortical neurones. J Neurochem . 2005;92:1386-1398.

14. Laufs U, Gertz K, Huang P, et al. Atorvastatin upregulates type III nitric oxide synthase in thrombocytes, decreases platelet activation, and protects from cerebral ischemia in normocholesterolemic mice. Stroke. 2000;31:2442-2449.

15. Sandercock P, Gubitz G, Foley P, Counsell C. Antiplatelet therapy for acute ischaemic stroke. Cochrane Database Syst Rev. 2003;(2):CD000029.

16. Iso H, Jacobs DRJ, Wentworth D, Neaton JD, Cohen JD. Serum cholesterol levels and six-year mortality from stroke in 350,977 men screened for the multiple risk factor intervention trial. $N$ Engl J Med. 1989;320:904-910.

17. Yano K, Reed DM, MacLean CJ. Serum cholesterol and hemorrhagic stroke in the Honolulu heart program. Stroke. 1989;20:1460-1465.

18. Ebrahim S, Sung J, Song YM, Ferrer RL, Lawlor DA, Davey Smith G. Serum cholesterol, haemorrhagic stroke, ischaemic stroke, and myocardial infarction: Korean national health system prospective cohort study. BMJ. 2006;333:22-28.

19. Leppala JM, Virtamo J, Fogelholm R, Albanes D, Heinonen OP. Different risk factors for different stroke subtypes: association of blood pressure, cholesterol, and antioxidants. Stroke. 1999;30:2535-2540.

20. Goldstein LB, Amarenco P, Szarek M, et al; on behalf of the SPARCL Investigators. Hemorrhagic stroke in the Stroke Prevention by Aggressive Reduction in Cholesterol Levels study. Neurology. 70: 2364-2370.

21. Amarenco P, Goldstein LB, Szarek M, et al; SPARCL Investigators. Effects of intense low-density lipoprotein cholesterol reduction in patients with stroke or transient ischemic attack: The Stroke Prevention by Aggressive Reduction in Cholesterol Levels (SPARCL) Trial. Stroke. 2007;38:3198-3204.

22. Amarenco P, Goldstein LB, Callahan A 3rd, et al; SPARCL Investigators Baseline blood pressure, low- and high-density lipoproteins, and triglycerides and the risk of vascular events in the Stroke Prevention by Aggressive Reduction in Cholesterol Levels (SPARCL) trial. Atherosclerosis. 2009;204:515-520.

23. Amarenco P, Goldstein LB, Messig M, et al; SPARCL Investigators. Relative and cumulative effects of lipid and blood pressure control in the Stroke Prevention by Aggressive Reduction in Cholesterol Levels trial. Stroke. 2009;40:2486-2492.

24. Baigent C, Keech A, Kearney PM, et al; Cholesterol Treatment Trialists' (CTT) Collaborators. Efficacy and safety of cholesterol-lowering treatment: prospective meta-analysis of data from 90056 participants in 14 randomised trials of statins. Lancet. 2005;366:1267-1278.

25. Rothwell PM, Coull AJ, Silver LE, et al. Population-based study of event-rate, incidence, case fatality, and mortality for all acute vascular events in all arterial territories (Oxford Vascular Study). Lancet. 2005;366:1773-1783.

26. Jemal A, Ward E, Hao Y, Thun M. Trends in the leading causes of death in the United States, 1970-2002. JAMA. 2005;294:1255-1259.

27. Sever PA, Dahlof B, Poulter NR, et al. Prevention of coronary and stroke events with atorvastatin in hypertensive patients who have average or lower-than-average cholesterol concentrations, in the Anglo-Scandinavian Cardiac Outcomes Trial-Lipid Lowering Arm (ASCOT-LLA): a multicentre randomised controlled trial. Lancet. 2003;361:1149-1158.

28. Shepherd J, Blauw GJ, Murphy MB, et al. Pravastatin in elderly individuals at risk of vascular disease (PROSPER): a randomized controlled trial. Lancet. 2002;360:1623-1630.

29. Lewis SJ, Moye LA, Sacks FM, et al. Effect of pravastatin on cardiovascular events in older patients with myocardial infarction and cholesterol levels in the average range. Ann Intern Med. 1998;129:681-689.

30. Ko DT, Mamdani M, Alter DA. Lipid-lowering therapy with statins in high-risk elderly patients: the treatment-risk paradox. JAMA. 2004;291:1864-1870.

31. Benner JS, Glynn RJ, Mogun H, Neumann PJ, Weinstein MC, Avorn J. Long-term persistence in use of statin therapy in elderly patients. JAMA. 2002;288:455-461. 
32. Chaturvedi S, Zivin JA, Breazna A, et al; for the SPARCL investigators. Effect of atorvastatin in elderly patients with a recent stroke or transient ischemic attack. Neurology. 2009;72:688-694.

33. Goldstein LB, Amarenco P, LaMonte M, et al. Relative effects of statin therapy on stroke and cardiovascular events in men and women: Secondary analysis of the Stroke Prevention by Aggressive Reduction in Cholesterol Levels (SPARCL) Study. Stroke. 2008;39:2444-2448.

34. Amarenco P, Benavente O, Goldstein LB, et al. Results of the Stroke Prevention by Aggressive Reduction in Cholesterol Levels (SPARCL) trial by stroke subtypes. Stroke. 2009;40:1405-1409.

35. Sillesen H, Amarenco P, Hennerici MG, et al; Stroke Prevention by Aggressive Reduction in Cholesterol Levels Investigators. Atorvastatin reduces the risk of cardiovascular events in patients with carotid atherosclerosis: a secondary analysis of the Stroke Prevention by Aggressive Reduction in Cholesterol Levels (SPARCL) trial. Stroke. 2008;39:3297-3302.

36. Executive Committee for the Asymptomatic Carotid Atherosclerosis Study. Endarterectomy for asymptomatic carotid stenosis. JAMA. 1995;273:1421-1428.

37. Halliday A, Mansfield A, Marro J, et al; MRC Asymptomatic Carotid Surgery Trial (ACST) Collaborative Group. Prevention of disabling and fatal strokes by successful carotid endarterectomy in patients without recent neurological symptoms: randomized controlled trial. Lancet. 2004;363:1491-1502.

38. Goldstein LB, Amarenco P, Zivin J, et al. Statin treatment and stroke outcome in the Stroke Prevention by Aggressive Reduction in Cholesterol Levels (SPARCL) Trial. Stroke. 2009;40:3526-3531.

39. Moonis M, Kane K, Schwiderski U, Sandage BW, Fisher M. HMG-CoA reductase inhibitors improve acute ischemic stroke outcome. Stroke. 2005;36:1298-1300.

40. Elkind MS, Flint AC, Sciacca RR, Sacco RL. Lipid-lowering agent use at ischemic stroke onset is associated with decreased mortality. Neurology. 2005;65:253-258.
41. Aslanyan S, Weir CJ, McInnes GT, Reid JL, Walters MR, Lees KR. Statin administration prior to ischaemic stroke onset and survival: exploratory evidence from matched treatment-control study. Eur $J$ Neurol. 2005;12:493-498.

42. Reeves MJ, Gargano JW, Luo Z, Mullard AJ, Jacobs BS, Majid A. Effect of pretreatment with statins on ischemic stroke outcomes. Stroke. 2008;39:1779-1785.

43. Karki K, Knight RA, Han Y, et al. Simvastatin and atorvastatin improve neurological outcome after experimental intracerebral hemorrhage. Stroke. 2009;40:3384-3389.

44. Lapchak PA, Han MK. The 3-hydroxy-3-methylglutaryl coenzyme A reductase inhibitor simvastatin reduces thrombolytic-induced intracerebral hemorrhage in embolized rabbits. Brain Res. 2009 15;1303:144-150.

45. FitzMaurice E, Wendell L, Snider R, et al. Effect of statins on intracerebral hemorrhage outcome and recurrence. Stroke. 2008;39:2151-2154.

46. Leker RR, Khoury ST, Rafaeli G, Shwartz R, Eichel R, Tanne D; NASIS Investigators. Prior use of statins improves outcome in patients with intracerebral hemorrhage: prospective data from the National Acute Stroke Israeli Surveys (NASIS). Stroke. 2009;40:2581-2584.

47. Amarenco P, Goldstein LB, Sillesen H, et al; Stroke Prevention by Aggressive Reduction in Cholesterol Levels Investigators. Coronary heart disease risk in patients with stroke or transient ischemic attack and no known coronary heart disease: findings from the Stroke Prevention by Aggressive Reduction in Cholesterol Levels (SPARCL) trial. Stroke. 2010;41:426-430.

48. Fitchett DH, Goodman SG, Langer A. Ischemic stroke: A cardiovascular risk equivalent? Lessons learned from the Stroke Prevention by Aggressive Reduction in Cholesterol Levels (SPARCL) trial. Can J Cardiol. 2008;24:705-708.

49. Amarenco P, Steg PG. Stroke is a coronary heart disease risk equivalent: implications for future clinical trials in secondary stroke prevention. Eur Heart J. 2008;29:1605-1607.
Vascular Health and Risk Management

\section{Publish your work in this journal}

Vascular Health and Risk Management is an international, peerreviewed journal of therapeutics and risk management, focusing on concise rapid reporting of clinical studies on the processes involved in the maintenance of vascular health; the monitoring, prevention and treatment of vascular disease and its sequelae; and the involvement of

\section{Dovepress}

metabolic disorders, particularly diabetes. This journal is indexed on PubMed Central and MedLine. The manuscript management system is completely online and includes a very quick and fair peer-review system, which is all easy to use. Visit http://www.dovepress.com/ testimonials.php to read real quotes from published authors. 